\title{
Hypervalent Iodine-Mediated Styrene Hetero- and Homodimerization Initiation Proceeds with Two-Electron Reductive Cleavage
}

\author{
Aqeel A. Hussein, ${ }^{1,2 *}$ Yumiao $\mathrm{Ma}^{3}$ and Ahmed Al-Yasari ${ }^{4,5}$ \\ ${ }^{1}$ School of Chemistry, University of Southampton, Southampton, Hampshire, SO17 1BJ, United Kingdom. \\ ${ }^{2}$ College of Dentistry, University of Al-Ameed, Karbala PO Box 198, Iraq. \\ ${ }^{3}$ BSJ Institute, Haidian, Beijing, 100084, People's Republic of China \\ ${ }^{4}$ School of Chemistry, University of East Anglia, Norwich, NR4 7TJ, United Kingdom. \\ ${ }^{5}$ Department of Chemistry, Faculty of Sciences, University of Kerbala, Kerbala, Iraq.
}

\begin{abstract}
A mechanistic insight into the hetero- and homodimerizations (HETD and HOMD) of styrenes promoted by hypervalent iodine reagents (HVIRs; DMP and PIDA) and facilitated by HFIP to yield all trans cyclobutanes is reported using density functional theory (DFT) calculations. The initialization involving direct bimolecular one-electron transfer is found to be highly unfavored, especially for the PIDA system. At this point, we suggest that the reaction is initiated with an overall two-electron reductive cleavage of two $\mathrm{I}-\mathrm{O}$ bond cleavages, affording I(III) (iodinane) and I(I) (iodobenzene) product with DMP and PIDA as oxidant, respectively. The resulting acetate groups are stabilized by the solvent HFIP through strong hydrogen bonding interaction, which promotes the electron transfer process. The nature of the electron transfer is studied in detail and found that the overall two-electron transfer occurs within tri-molecular complex organized by $\pi$-stacking interactions and as a stepwise and concerted mechanism for I(III) and I(V) oxidants, respectively. The reaction rate is determined by the initialization step: for I(III), the initiation is thermodynamically endergonic, whereas the endergonicity for $\mathrm{I}(\mathrm{V})$ is modest. Upon initialization, the reaction proceeds through a stepwise [2+2] pathway, involving a radicalcationic $\pi-\pi$ stacked intermediate, either hetero- or homodimerized. DFT results supported by quasiclassical molecular dynamics simulations show that HOMD is dynamically competing pathway to HETD although the latter is relatively faster, in accordance with experimental observations.
\end{abstract}




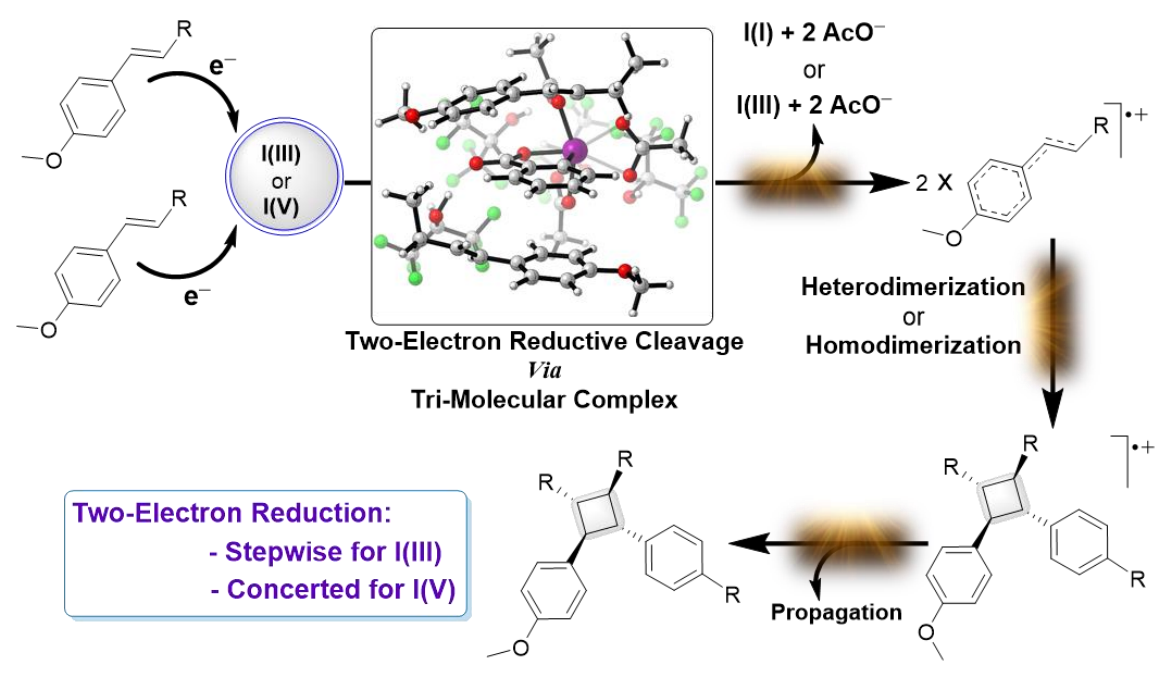

\section{Introduction}

Stereoselective approaches to substituted cyclobutanes have been captivated by organic chemists to be of high interest despite of its challenging requirements. ${ }^{1,2}$ Due to the fact that these cyclobutane rings exist in many bioactive natural products (Figure 1-a), ${ }^{3-5}$ the need for such efficient, reliable, and benign synthesis methods is still under developing strategies to get a purely chiral strained carbocycle. Regardless the many different synthetic methodologies appeared in literatures to access cyclobutanes, ${ }^{6-11}$ the olefin dimerization via oxidative manners, which involves an active radical cation intermediate formation, represents a helpful and promising tactic to reach. The olefin dimerization was firstly reported by Ledwith ${ }^{12,13}$ and Bauld, ${ }^{14-16}$. In this regard, metal complexes ${ }^{17}$ and organic ${ }^{18,19}$ photoredox catalysis have been applied to promote such a nice cyclization. ${ }^{20-28}$ Recently, a major contribution to this field has been exploited by using catalytic amounts of HVIR ${ }^{29,30}$ in HFIP to investigate a stereoselective functionalization of alkenes. ${ }^{31-33}$ The HFIP has been shown to be a unique solvent due its significant role of hydrogen bonding ${ }^{34-36}$ that enables the HVIR to act as single electron oxidants. ${ }^{37-40}$ Based on the utility of the HVIR/HFIP, Donohoe and co-workers have developed a diastereoselective [2+2] cycloaddition of alkenes with remarkable results (Figure 1-b). ${ }^{41,42}$ The mechanism proposed involves a SEO of styrene $\mathbf{1}$ to a radical cation $\mathbf{1}^{+}$by HVIR followed by either HOMD, where dimerization proceeds with another molecule of styrene $\mathbf{1}$ in the presence of I(III) phenyliodine diacetate (PIDA), or HETD, where dimerization proceeds with a different alkene $\mathbf{2}$ in the presence of I(V) Dess-Martin periodinane (DMP), to give the all trans cyclobutane product 3 after the re-addition of an electron to the product. The presence of a $p$-methoxy group plays an important role in the success of a styrene toward dimerization. 
a) Bioactive natural products:<smiles>O=C(C1/C(=C/c2ccc3c(c2)OCO3)[C@H](/C=C/c2ccc3c(c2)OCO3)C1C(=O)N1CCCCC1)N1CCCCC1</smiles>

Nigramide R<smiles>O=C(Nc1ccccc1)C1C(C(=O)Nc2ccccc2)C(c2ccccc2)C1c1ccccc1</smiles>

$\zeta$-Truxinanilide

b) Iodine-mediated dimerization of Styrenes:<smiles>[R]C=Cc1ccc(OC)cc1</smiles>

$\mathrm{F}_{3} \mathrm{C}$

I(III) (PIDA) or I(V) (DMP)<smiles>[R]C1=C([R])C(c2ccc(OC)cc2)=C1[Y]</smiles>

HFIP, $\quad A r \sim / R, 12 \mathrm{~h}$

2

up to $95 \%$ yield, d.r. $\geq 95: 5$

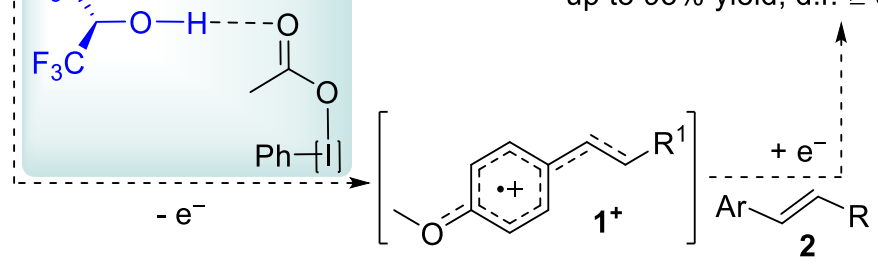

Figure 1. a) Examples of bioactive natural products containing cyclobutane ring. b) HOMD or HETD of styrenes mediated with PIDA or DMP, respectively .

The existence of hydrogen bonding interactions between the HFIP and PIDA has been proposed to be essential and the physical origin of the enhanced oxidative strength for the iodine reagent. ${ }^{43}$ In addition to the almost disappearance of HO signals from NMR experiments, the voltammetric peak potential experiments measured versus $\mathrm{Fc} / \mathrm{Fc}^{+}$demonstrated a shift in reduction potentials for PIDA $\left(E_{\mathrm{p}, \mathrm{c}}\right.$ in $\mathrm{ACN}=-1.32 \mathrm{~V}, E_{\mathrm{p}, \mathrm{c}}$ in $\mathrm{HFIP}=$ -0.47 V). The possibility of ligand exchange between HFIP and PIDA has been excluded and any altered reactivity to the oxidants is ruled out as the HFIP is a low nucleophilic solvent. ${ }^{43-47}$ All of the above-mentioned study concerns the first step of the reaction, the SEO step, and seems to us in need for further understandings despite the subsequent steps that lead to the all trans cyclobutane ring are not considered, at least to the best of our knowledge, by other workers under these conditions. ${ }^{26,48-50}$ An important question that should be raised is the number of electrons to be transferred to the iodine reagent to initiate the reaction. At this point, the reaction mechanism and reactivity of HVIR-mediate dimerization exclusively appears incomplete and warrants further attentions (Figure 2). Therefore, we herein interpret DFT simulations on the HOMD and HETD that gives all 
trans cyclobutane under HVIRs with PIDA and DMP, respectively, featuring (1) the nature of initiation whether one or two electron reduction with considerations involved the effect of HFIP on reactivity of this protocol, and (2) realizing the dynamical nature of homo- and heterodimerization via quasiclassical trajectory molecular dynamics (QCTMD) simulations. ${ }^{51}$

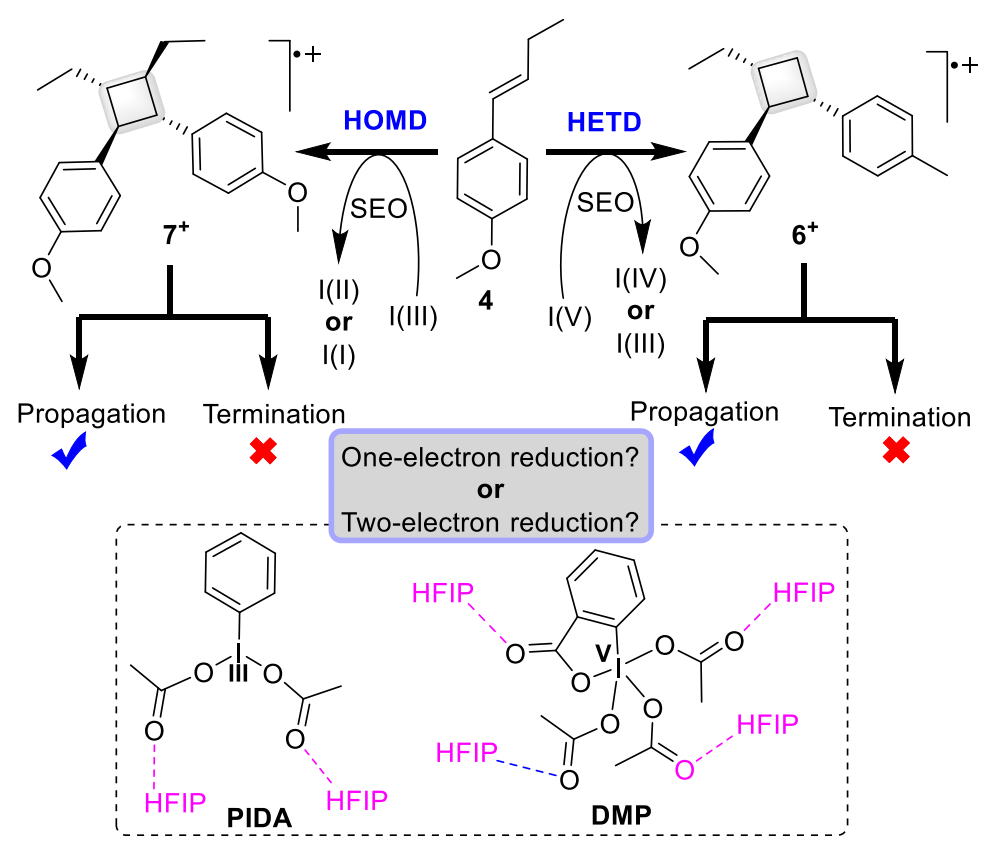

Figure 2. General representation of the HVIR [2+2] cycloaddition considered in this study, where iodine reagents are hydrogen bonded to HFIP explicitly.

\section{Results and Discussion}

To explore our HVIR-mediated dimerization of styrenes, we have divided our discussions into five distinct sections with the following order: validation of our strategy, mechanism of dimerization, molecular dynamics of HOMD and HETD.

\section{Validation of strategy and level of theory}

The calculations were conducted in explicit and implicit HFIP. The explicit HFIP protocol means that every single acetate groups in PIDA and DMP is hydrogen bonded to one HFIP molecule to match the experimental conditions, whereas the implicit protocol is performed only with continuum solvation model based on density 
(IEFPCM-SMD). All structures were initially calculated using the $w \mathrm{~B} 97 \mathrm{XD} / 6-311+\mathrm{G}(\mathrm{d}, \mathrm{p}) / \mathrm{LANL} 2 \mathrm{DZ} / / 6$ 31G(d)/LANL2DZ level of theory, however we found inconsistencies with the experimental results because of Fe and I atoms. Therefore, we carried out a basis set search on Fe and I atoms through running single point energy calculations on optimized structure by 6-31G(d)/LANL2DZ through comparison between measured and calculated voltammetric peak potentials for redox species of different substituted trans- $\beta$-methylstyrenes toward PIDA (Figure 10, see below). For $\mathrm{Fc} / \mathrm{Fc}^{+}$, as shown in SI, the cyclopentadienyl group in $\mathrm{Fc}$ was tested with a basis set of triple- $\zeta$ quality $(6-31 \mathrm{G}(\mathrm{d}, \mathrm{p}))$ to be consistent with the valence basis sets used for iron. We found that Def2-TZVPP/6-31G(d,p) level of theory gives the best agreement with the experimental redox potential values of different substituted trans- $\beta$-methylstyrenes using $\mathrm{Cp}_{2} \mathrm{Fe}$ (calculated $E^{1 / 2}=4.84 \mathrm{~V}$ ) as reference to calculate their redox potentials (see Figure 10). For iodine in PIDA, it was found that the basis set Def2-TZVPP for iodine and $6-311+\mathrm{G}(\mathrm{d}, \mathrm{p})$ for $\mathrm{C}, \mathrm{H}$, and $\mathrm{O}$ atoms gave the best agreement with experimental redox potentials (see SI). Importantly, and under explicit protocol, the calculated value for PIDA $_{\text {HFIP }}+\mathrm{e}^{-} \rightarrow$ PIDA $_{\text {HFIP is }}^{-1 / 2}$ $=4.25 \mathrm{~V}$ of peak potential for PIDA HFIP $_{\text {is }} E_{\mathrm{p}, \mathrm{c}}=-0.59 \mathrm{~V}$ versus calculated peak potential $\mathrm{Fc} / \mathrm{Fc}^{+}\left(E^{1 / 2}=4.84\right.$ $\mathrm{V}$ ) as a reference, leading to a good agreement with the measured peak potential for PIDA is $E_{\mathrm{p}, \mathrm{c}}=-0.47 \mathrm{~V}$. Under implicit protocol, the calculated value of non-hydrogen-bonded PIDA is $E^{1 / 2}=4.02 \mathrm{~V}$ of peak potential $E_{\mathrm{p}, \mathrm{c}}=-0.82 \mathrm{~V}$ versus $\mathrm{Fc} / \mathrm{Fc}^{+}$with a shifting to more negative value of $230 \mathrm{mV}$ less favorable than explicit PIDA $_{\text {HFIP. Using this strategy, a good agreement between the measured and calculated peak potential have been }}$ achieved as shown in Figure 10. Therefore, the $w$ B97XD/def2-TZVPP/6-311+G(d) level of theory is used for oxidants whereas the $w \mathrm{~B} 97 \mathrm{XD} / \mathrm{def} 2-\mathrm{TZVPP} / 6-31 \mathrm{G}(\mathrm{d}, \mathrm{p})$ level of theory is utilized for $\mathrm{Cp}_{2} \mathrm{Fe}$ in order to calculate the redox potentials. Comparison between calculated and measured redox potentials of different substituted trans- $\beta$-methylstyrenes $\mathbf{4 a}-\mathbf{4 k}$ is indicated in Figure 3. Our strategic DFT simulations present a very good agreement with the experimental redox potentials and free energy of reoxidation accordingly. The explicit-involved HFIP calculations are consistent with experimental results than inexplicit calculations (for comparison see SI). The calculated results appeared in Figure 3-b indicate a deviation from experimental values of around $0.12 \mathrm{eV}$ which is in agreement with the mean absolute error in ionization energy $\left(2.74 \mathrm{kcal} \mathrm{mol}^{-1}\right)$ reported for $w \mathrm{~B} 97 \mathrm{XD} .^{52}$ 
a)

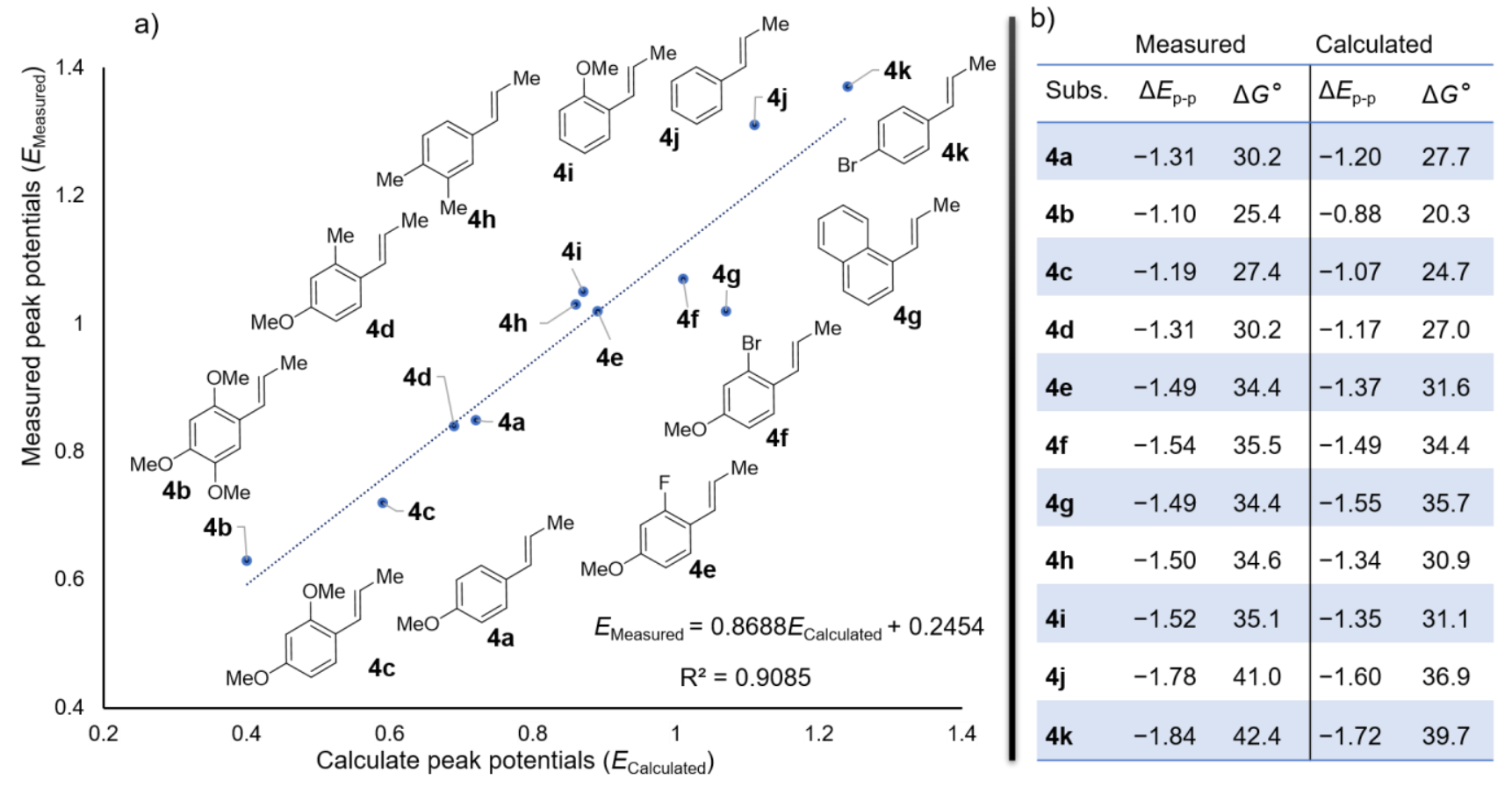

Figure 3. (a) Agreement between measured and calculated voltammetric peak potentials (in V) for redox species of different substituted trans- $\beta$-methylstyrenes $\mathbf{4 a}-\mathbf{4 k}$. (b) Differences in the reduction and oxidation peak potentials (in V) and their Gibbs free energies (in $\mathrm{kcal} \mathrm{mol}^{-1}$ ) for styrenes $\mathbf{4 a}-\mathbf{4} \mathbf{k}$. Styrenes $\mathbf{4 g}-\mathbf{4} \mathbf{k}$ did not undergo PIDA [2+2] cycloaddition. The measured results were obtained versus $\mathrm{Fc} / \mathrm{Fc}^{+}$, as measured at $100 \mathrm{mV}$ $\mathrm{s}^{-1} \cdot{ }^{43}$ The calculated $\mathrm{Fc} / \mathrm{Fc}^{+}$is $E^{1 / 2}=4.84 \mathrm{~V}$ in HFIP. The calculated value for PIDA $\mathbf{H F I P}+\mathrm{e}^{-} \rightarrow$ PIDA $^{-}$HFIP is $E^{1 / 2}=4.25 \mathrm{~V}$. Measured peak potential for PIDA is $E_{\mathrm{p}, \mathrm{c}}=-0.47 \mathrm{~V} \cdot{ }^{43}$ Th calculated peak potential for PIDA $\mathbf{H F I P}_{\mathrm{P}}$ $E_{\mathrm{p}, \mathrm{c}}=-0.59 \mathrm{~V}$.

\section{Mechanism of dimerization}

General Considerations. Our DFT investigations with the exploration of the HETD and HOMD facilitated by DMP and PIDA, respectively, are considered. Firstly, the cyclobutane ring formation is investigated based on the SEO and single electron reduction (SER). When the SEO and SER are initiated and terminated, respectively, through only HVIR to get cyclobutane ring formed, this is a catalytic mechanism. The more plausible scenario is that the HVIR only initiates the reaction to get the styrene molecule radicalized by SEO and propagation of the reaction proceeds without HVIR and this is an initiated or propagated mechanism as the HVIR serves as an initiator. Secondly, to account better knowledge about the height barrier of SEO, free energy of activation for 
the SEO was calculated using four-point method proposed by Nelsen (see SI). ${ }^{53-55}$ We are convinced that this method result in a reasonable estimation of the electron transfer (ET) activation barrier. Thirdly, it has been reported that HFIP plays a critical role with oxidizing agent rather than with the radical cation formed. ${ }^{43}$ The effect of explicit hydrogen bonding in our calculations is considered only on the SEO steps, whereas the cyclization steps are proceeded with an implicit HFIP protocol.

HETD pathway. The DFT results of HETD in the presence I(V) DMP with and without explicit HFIP molecules have been exploited (for comparison between explicit and implicit HFIP see SI). Initially, the iodine catalyst undergoes either one-electron reduction to give I(IV) or two-electron reduction to give I(III), namely iodinane. Both pathways are investigated and shown in Figure 4. On one hand, when the initiation proceeds with a one-electron process, single electron transfer from one styrene to $\mathrm{I}(\mathrm{V})$, the free energy of activation for SEO for the FRS was found to be $20.2 \mathrm{kcal} \mathrm{mol}^{-1}$ to give radical cation $\mathbf{4}^{+}$and radical anion DMP $^{-}$HFIP as an endergonic step $\left(\Delta \mathrm{G}_{\mathrm{r}}=13.8 \mathrm{kcal} \mathrm{mol}^{-1}\right)$ (Figure 4). In absence of explicit HIFP the barrier for SEO increased to $25.4 \mathrm{kcal} \mathrm{mol}^{-1}$ as a more endergonic process $\left(\Delta \mathrm{G}_{\mathrm{r}}=21.8 \mathrm{kcal} \mathrm{mol}^{-1}\right)($ See SI). An apparent increased in the I-O bond distances, clearly represented for the perpendicular acetate units to the phenyl iodine. After the ET, the I-O bond distances elongate from 2.08 and $2.15 \AA$ to $2.63 \AA$ and $2.99 \AA$ when HFIP are not involved in calculations explicitly (see DMP and DMP- in Figure S3). Elongation is slightly less when HFIP is involved explicitly, where I-O bond length is $2.12 \AA$ is before the SEO and $2.55 \AA$ and $2.77 \AA$ are after the SEO (see DMP $_{\text {HFIP }}$ and DMP ${ }_{\text {HFIP }}^{-}$in Figure 4). On the other hand, a lower and more favored energetic pathway was found when a two-electron reduction process is involved, accompanying by two I-O bond cleavages, occurring through two SEOs from two styrenes give iodinane I(III) and two acetate groups stabilized by strong hydrogen bonding interactions (see Iodinane $\mathbf{2} \mathbf{A c O}^{-}$HFIP in Figure 4). This pathway is lower than one-electron pathway by more than $7.0 \mathrm{kcal} \mathrm{mol}^{-1}$. Here, addition of two electrons from two styrenes found to need only $5.4 \mathrm{kcal}$ $\mathrm{mol}^{-1}$ as a free energy of reduction. Similarly, the change in oxidation state $\mathrm{I}(\mathrm{V}) \rightarrow \mathrm{I}(\mathrm{III})$ has been reported for oxidation of alcohols to give iodinane and two acetic acid molecules. ${ }^{56-58}$ A good support to the two-electron reduction comes from the favorable formation of tri-molecular complex between two styrene molecules and iodine reagent (see below).

All trails to find a concerted [2+2] cycloaddition TS for the cation cyclobutane formation $6^{+}$are unsuccessful and, therefore, a two-step mechanism have been taken through the stepwise cycloaddition. For the first C-C 
bond formation, the head-to-head first $\mathrm{C}-\mathrm{C}$ bond formation was found to have a barrier of $8.3 \mathrm{kcal} \mathrm{mol}^{-1}$ via TS $8^{+}$with bond length of $2.20 \AA \AA$ along the TS is established, giving uncyclized intermediate $\mathbf{9}^{+}$with $\mathrm{C}-\mathrm{C}$ bond being formed at $1.58 \AA$ as a thermoneutral step of $0.1 \mathrm{kcal} \mathrm{mol}^{-1}$ (Figure 3 ). ${ }^{59}$ The TS $\mathbf{8}^{+}$shows a $\pi-\pi$ stacking interaction of 3.6 $\AA$. A higher barrier TS of $13.1 \mathrm{kcal} \mathrm{mol}^{-1}$ was found without $\pi$ - $\pi$ stacking (see Figure S2). It seems that the favorable $\pi$ - $\pi$ stacking plays an important role in controlling the configurations of the product to be all trans cyclobutane. The presence of non-covalent interaction, $\pi-\pi$ stacking, for TS $\mathbf{8}^{+}$and intermediate $\mathbf{9}^{+}$ is shown by Reduced Density Gradient (RDG) analysis (see Figure S6) ${ }^{60}$ Attractive $\pi-\pi$ interaction is clearly seen in the green areas between the two phenyl rings. The nature of interaction between $\mathbf{4}^{+}$and $\mathbf{5}$ through TS $\mathbf{8}^{+}$ has a radical character due to SOMO-HOMO overlapping. The SOMO orbitals located on radical styrene $\mathbf{4}^{+}$is overlapped with the HOMOs of 5 with an energy gap if $4.03 \mathrm{eV}$ (see Figure S11). The radical cation intermediate $\mathbf{9}^{+}$cyclizes to the cationic cyclobutane $\mathbf{6}^{+}$in a low barrier step of $\Delta \mathrm{G}^{\ddagger}=7.1 \mathrm{kcal} \mathrm{mol}^{-1}$ with a long $\mathrm{C}-\mathrm{C}$ bond of $2.74 \AA$ along TS $\mathbf{1 0}^{+}$but in a slightly exergonic step $\left(\Delta \mathrm{G}_{\mathrm{r}}=-2.1 \mathrm{kcal} \mathrm{mol}^{-1}\right)$. Noticeably, the new $\mathrm{C}-\mathrm{C}$ bond formed in cyclobutane $\mathbf{6}^{+}$is $1.64 \AA$ whereas all other $\mathrm{C}-\mathrm{C}$ bonds in the ring are $1.54 \AA$, and this is attributed to radical character as indicated by the partial delocalization shown by spin density and $\beta$-LUMO contours (see Figure S13). To release the cyclobutane $\mathbf{6}$, the radical cation $\mathbf{6}^{+}$undergoes SER by or another styrene to propagate the reaction. The oxidation of styrene 4 by $\mathbf{6}^{+}$is nearly to be barrierless of $1.1 \mathrm{kcal} \mathrm{mol}^{-1}$ as an exergonic step $\left(\Delta \mathrm{G}_{\mathrm{r}}=-6.6 \mathrm{kcal} \mathrm{mol}^{-1}\right)($ Figure 4$)$. 


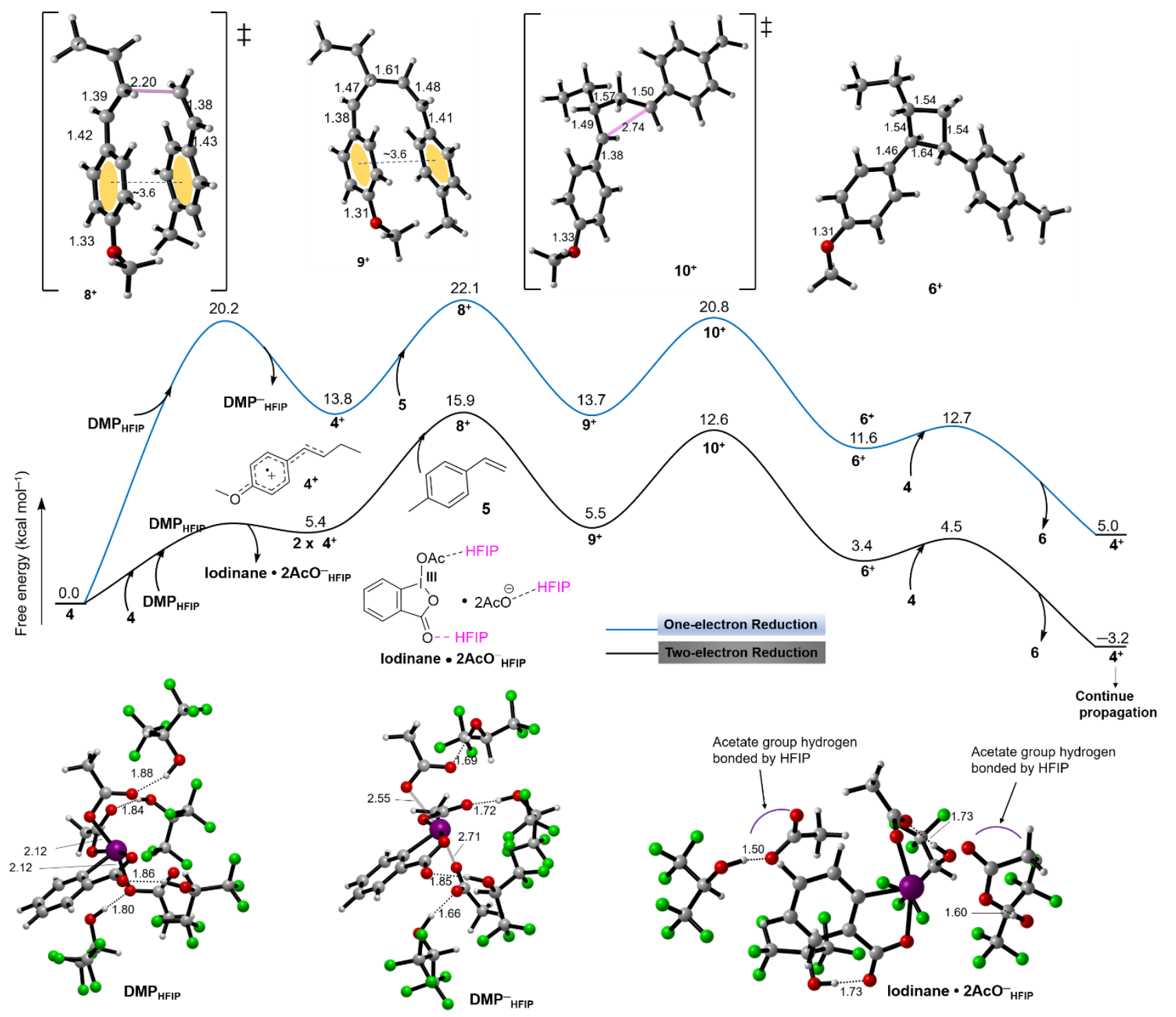

Figure 4. Free energy profile for the mechanism of DMP-mediated heterodimerization of styrenes (4) and (5) to yield cyclobutane 6 under one-electron reduction (blue pathway) and two-electron reduction (black pathway) hydrogen-bonded with HFIP. Bond lengths are in $\AA$.

HOMD pathway. Following the same strategy for HETD, the HOMD mechanism in the presence I(III) PIDA is investigated and shown in Figure 5. Under single electron reductive initiation, the barrier of SEO, the FRS, was found to be $31.3 \mathrm{kcal} \mathrm{mol}^{-1}$ to give radical cation $\mathbf{4}^{+}$and anion $\mathbf{P I D A}^{-}$HFIP as an endergonic step $\left(\Delta \mathrm{G}_{\mathrm{r}}=\right.$ $28.1 \mathrm{kcal} \mathrm{mol}^{-1}$ ). The SEO for HOMD is more endergonic than for the HETD. The calculated endergonicity for

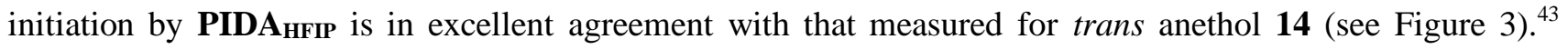
Following the SEO step the bond length of the acetate group to iodine, namely I-O bonds, increases from 2.15 
$\AA$ to around $2.58 \AA$ for the non-hydrogen bonded PIDA (Figure S4) and to longer distances of $2.66 \AA$ and 2.78 $\AA$ for the hydrogen-bonded one PIDAHFIP (Figure 5) for the reason mentioned above for SEO by DMP PFIP. $_{\text {. }}$ However, when a reductive cleavage process, two-electron reduction, iodobenzene and two acetate groups stabilized by HFIP (Iodobenzene $\cdot \mathbf{2 A c O}^{-}$HFIP) as well as two cationic styrenes are produced in a less endergonicity step of $21.4 \mathrm{kcal} \mathrm{mol}^{-1}$. Comparison of one and two electron process initiations, the impact is substantially effective for PIDA-HOMD protocol in comparison to DMP-HETD protocol. The synthetic utility with PIDA/HFIP is considered to be mild conditions and the one-electron reduction would be highly unlikely and, therefore, two-electron process is required to initiate the radically-cationic $[2+2]$ cycloaddition reaction. Reported literatures have shown that I(III), PIDA, undergoes a reductive cleavage of their I-O bonds under to yield the corresponding I(I), namely iodobenzene. ${ }^{61}$ This has been also reported for oxidative of alcohols. ${ }^{44}$ The process for $\mathbf{4}^{+} \rightarrow \mathbf{1 2}^{+}$has a reasonable barrier of $9.5 \mathrm{kcal} \mathrm{mol}^{-1}$ via $\pi-\pi$ stacked head-to-head TS $\mathbf{1 1}^{+}$with bond length of $2.16 \AA$ to give the cationic uncyclized intermediate $\mathbf{1 2}^{+}$as an endergonic step of $4.3 \mathrm{kcal} \mathrm{mol}^{-1}$ (Figure 5). ${ }^{59}$ The favorable $\pi-\pi$ stacking interaction between the two phenyl rings is shown in Figure S6. A higher barrier TS of $12.9 \mathrm{kcal} \mathrm{mol}^{-1}$ was found for the first $\mathrm{C}-\mathrm{C}$ bond formation when aromatic rings are not stacked (Figure S2). Likely to TS $\mathbf{8}^{+}$, TS $\mathbf{1 1}^{+}$has a radical character with an energy gap of $4.86 \mathrm{eV}$ (Figure S12) which is higher than for the HETD $\left(\mathbf{4}^{+}\right.$and $\left.\mathbf{5}\right)$. The first $\mathrm{C}-\mathrm{C}$ bond formation in $\mathbf{1 2}^{+}$is longer than for that found for the uncyclized heterodimerized intermediate $\mathbf{9}^{+}$. The cyclization, TRS, is a low barrier step of $4.3 \mathrm{kcal} \mathrm{mol}^{-1}$ through $\mathrm{TS} \mathbf{1 3}^{+}$with $\mathrm{C}-\mathrm{C}$ bond at $2.16 \AA$ is seen to give the cationic homodimerized cyclobutane $\mathbf{7}^{+}$as an exergonic step $\left(\mathbf{1 2}^{+} \rightarrow \mathbf{7}^{+}, \Delta \mathrm{G}_{\mathrm{r}}=-5.3 \mathrm{kcal} \mathrm{mol}^{-1}\right.$, Figure 5). Upon formation of $\mathbf{7}^{+}$, the unpaired electron has totally delocalized over the entire system of $7^{+}$(see Figure S13) and resulted in an increase in the new $\mathrm{C}-\mathrm{C}$ bond to be $1.71 \AA$, being longer than for $\mathbf{6}^{+}$. The release of neutral homodimerized cyclobutane 7 via propagation process (Figure 5) is calculated to be kinetically and thermodynamically favored. The oxidation of styrene $\mathbf{4}$ by $7^{+}$is found to be nearly barrierless of $1.5 \mathrm{kcal} \mathrm{mol}^{-1}$ as an exergonic step $\left(\Delta \mathrm{G}_{\mathrm{r}}=-6.3 \mathrm{kcal} \mathrm{mol}^{-1}\right)$ in order to propagate the reaction. 


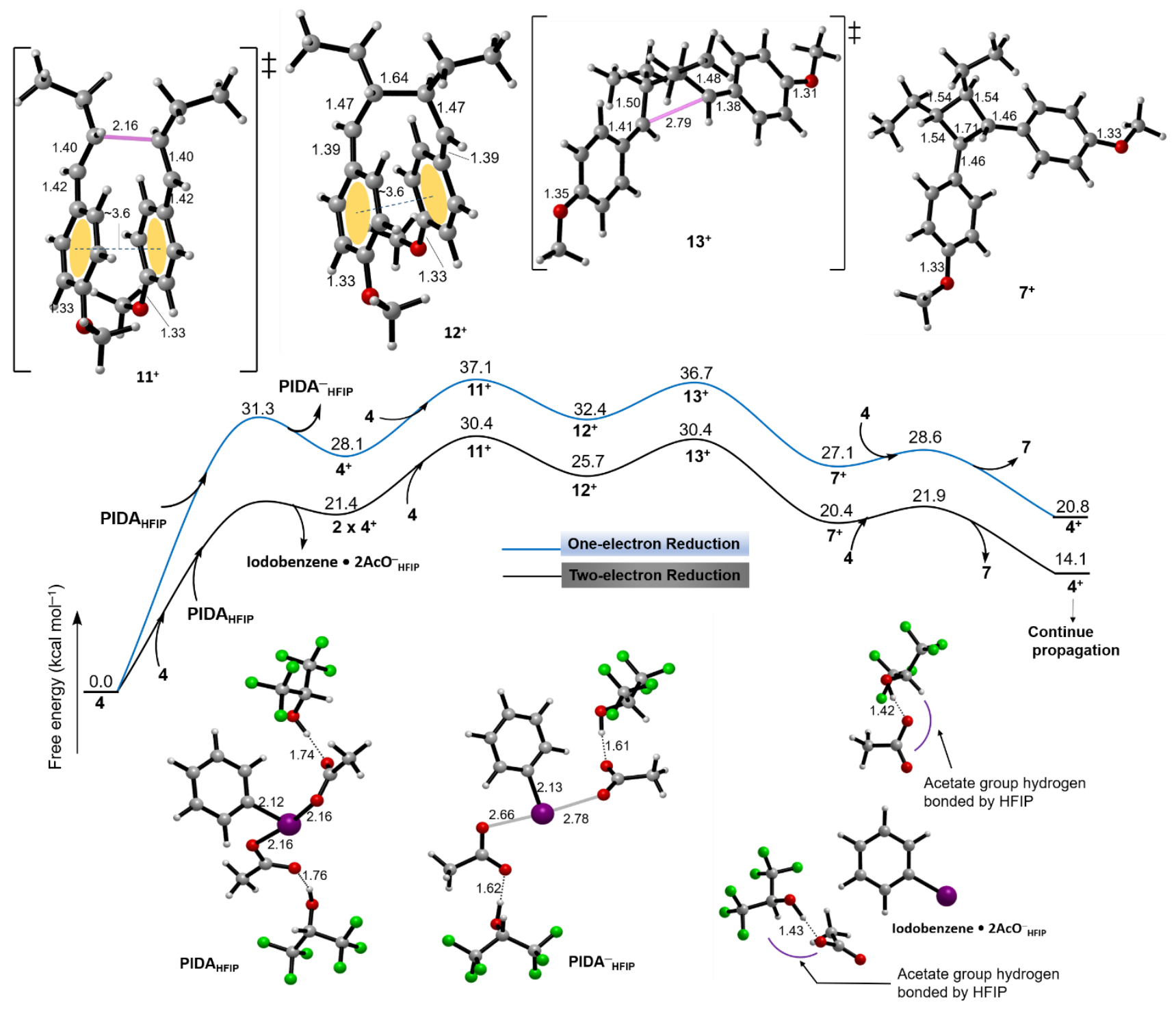

Figure 5. Free energy profile for the mechanism of PIDA-mediated heterodimerization of styrenes (4) to yield cyclobutane 7 under one-electron reduction (blue pathway) and two-electron reduction (black pathway) hydrogen-bonded with HFIP. Bond lengths are in $\AA$. 
Validity of two-electron oxidation mechanism. More detailed investigation on the overall two-electron oxidation was conducted, due to its key role in the initialization step as well as its uncommon and unexplored nature in solution. The main aim of this section is to clarify two questions: (1) how could the seemingly entropically unfavored 2e-transfer be possible? and (2) is the overall 2e-transfer concerted or stepwise? It is found that (1) the overall 2e-transfer is enabled by the thermodynamically favored formation of a tri-molecular complex organized by pi-stacking; (2) PIDA and DMP follow different reaction pathway for the overall 2etransfer. The first concern about the 2e-transfer is that it demands the formation of a tri-molecular complex, which is uncommon and entropically unfavored at the first glance. However, the trimer formation is shown to be rather favorable (Figure 6). The Gibbs free energy for the trimer was determined to be +0.4 and $-3.6 \mathrm{kcal}$

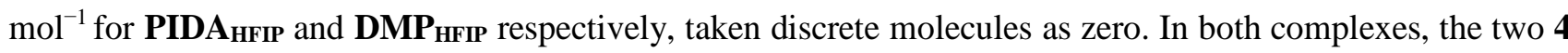
molecules parallel the phenyl ring plane of the electron-deficient HVIR with their electron-rich $\pi$-system. The $\pi$-stacking interactions are believed to provide stabilization to the trimer complexes, which is further depicted by the RDG analysis. The preorganization in the trimer complexes is believed to promote further electron transfer.

The favorable formation of trimer complex encouraged us to further examine the 2e-transfer process, especially its concertedness. Huge efforts were paid to locate transition states, especially for elementary steps involving potential concerted acetate dissociation-electron transfer process. Unfortunately, it was proven rather difficult. Instead, the energetics of several related intermediates were examined. These intermediates were obtained by plenty of geometry optimization calculations with various initial geometries, in a broken-symmetry selfconsistent field (BS-SCF) manner. While the trimer complex without electron transfer is in its close-shell state, the 1e-transfer (1ET) and 2e-transfer (2ET) product should exhibit an open-shell singlet (or triplet) state and can be distinguished from their spin density distribution. By studying their geometry and energetics, one can obtain some insight into the nature and process of the overall 2e-transfer. 


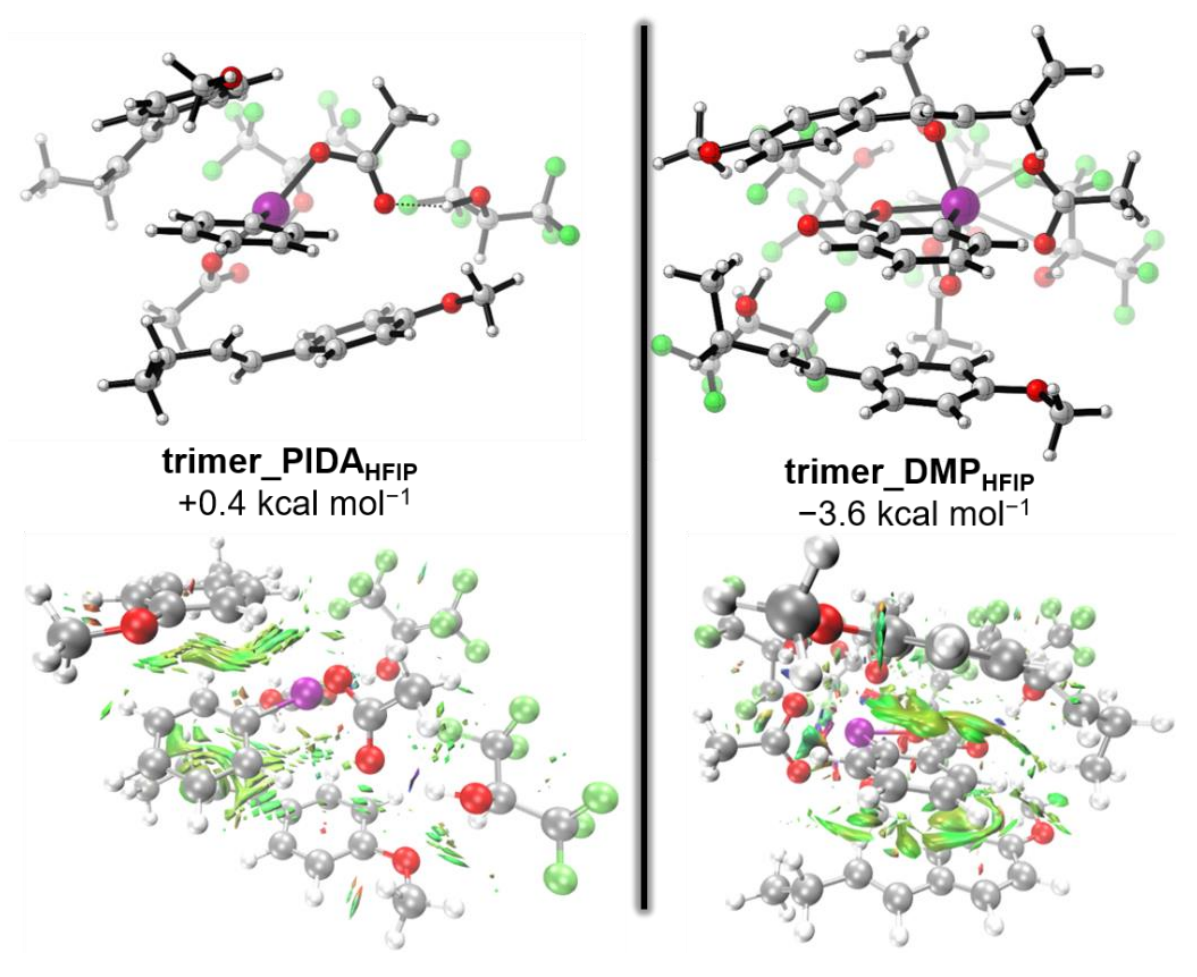

Figure 6. The geometry (up) and the RDG isosurface (down) for the trimolecular complexes formed by two

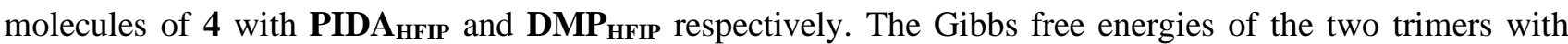
discrete molecules as zero point.

Figure 7 show the geometries and spin density of the possible intermediates for the overall 2e-transfer between DMP $_{\text {HFIP }}$ and 4. No open-shell singlet states were found nearby the trimer_DMP HFIP $_{\text {geometry, and thus it is }}$ believed that the electron transfer is accompanied by the dissociation of one acetate ligand. It is clear from the I-O distance that the equatorial acetate group binds more weakly with the iodine center than its axial analogues. Indeed, on the contrary to the axial acetate cleavage which is $34.1 \mathrm{kcal} \mathrm{mol}^{-1}$ endergonic, the dissociation of the equatorial acetate is slightly exothermic by $-2.8 \mathrm{kcal} \mathrm{mol}^{-1}$, and directly affords an open-shell singlet product

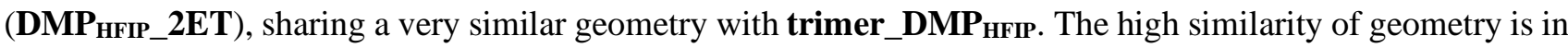
agreement with a concerted 2e-transfer. The spin density isosurface clearly shows that both of the two 4 subunits are single-electron oxidized, indicating that the 2e-transfer event has been accomplished for DMP $\mathbf{P}_{\mathbf{H F I P}} \mathbf{2 E T}$. On

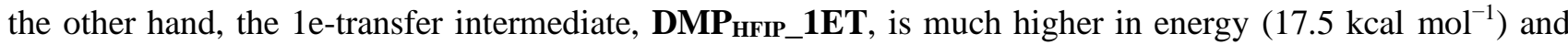
bears large geometrical reorganization compared to trimer_DMP $\mathbf{P}_{\text {HFIP. }}$ Overall, the present results strongly agree with a concerted 2e-transfer accompanied by the dissociation of one equatorial acetate ligand for $\mathbf{D M P}$ HFIP, 


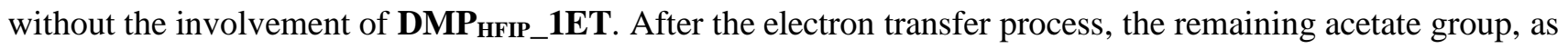
well as the two 4+ cations, is expected to dissociate and diffuse into the solution, affording the iodinane intermediate as indicated in Figure 4.
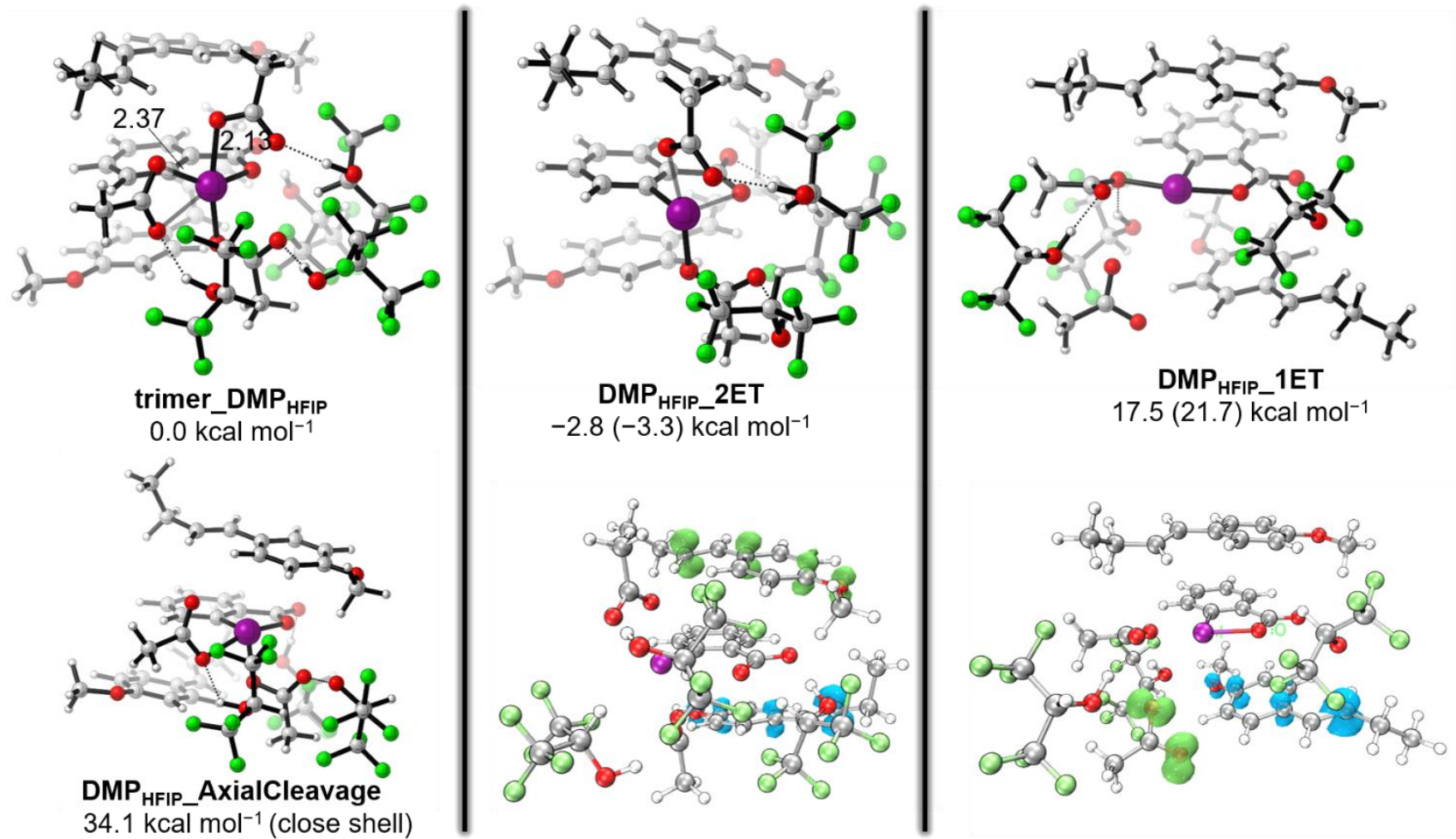

Figure 7. The geometries and spin density isosurfaces (with isovalue of 0.01) of the possible intermediates for the overall 2e-transfer between DMP HFIP $_{\text {and }}$ 4. Energies for the triplet state are shown in brackets. Bond lengths are in $\AA$.

The situation is different for the trimer formed by PIDA $\mathbf{H F I P}_{\text {and }} \mathbf{4}$ (Figure 8). On the contrary of DMP $\mathbf{P}_{\mathbf{H F I P}}$, the 2e-transfer product, PIDA_HFIP_2ET, has a significantly different geometry from trimer_PIDAHFIP. For this complex, the two $4^{+}$subunits are linked by the two acetate anions through electrostatically favored $\mathrm{CH}-\mathrm{O}$ hydrogen bonding, and the $\mathrm{PhI}$ fragment is excluded from its original position. In addition, the two 4 subunits need to rotate to reach its position in PIDAHFIP_2ET from trimer_PIDAHFIP. No electron-transferred states were found nearby the trimer_PIDA transfer could not happen in one elementary step. The dissociation of the acetate ligand was also examined, but no 2e-transfer product could be located, further precluding the involvement of a DMP-like concerted I-O dissociation-2e-transfer process. As a result, we suggest the following stepwise process. The trimer_PIDAHFIP 


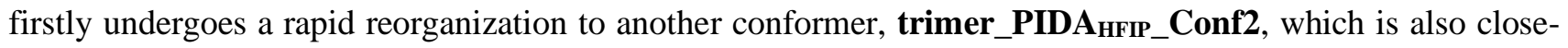
shell in nature. The latter undergoes an intramolecular 1e-transfer to afford the PIDAHFIP_1ET complex with minor geometry reorganization, for which the spin density is distributed over the AcO-I-OAc substructure. Upon the further elongation of the two I-OAc bonds, the second electron transfer happens between the O-I-O structure and the remaining unoxidized 4 subunit, finally affording the PIDAHFIP_2ET product.

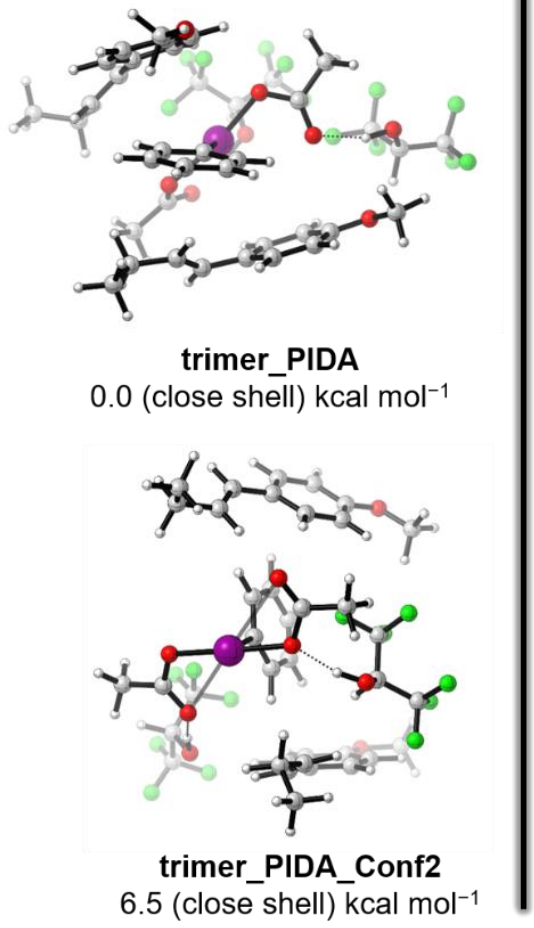

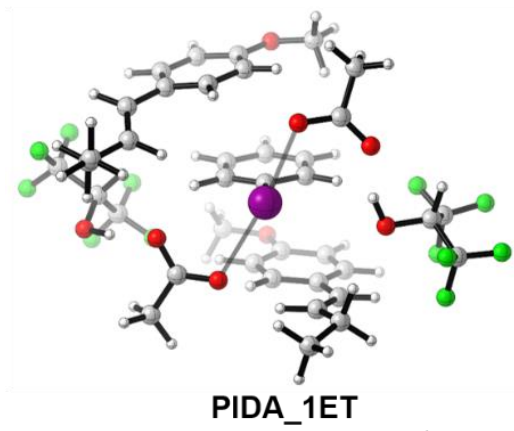

$24.3(24.4) \mathrm{kcal} \mathrm{mol}^{-1}$

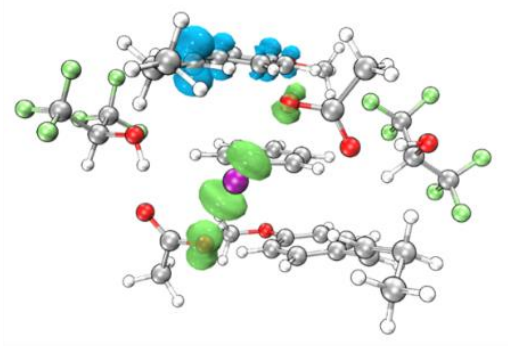

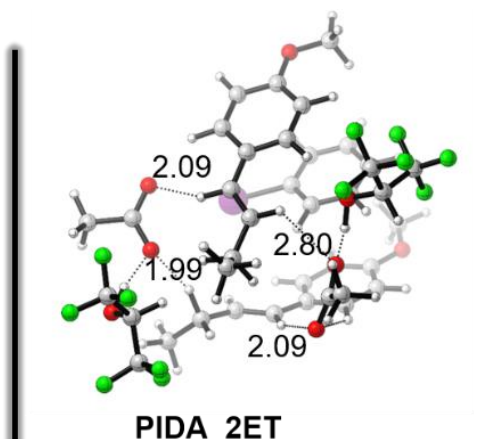

PIDA 2ET

$15.4(14.5) \mathrm{kcal} \mathrm{mol}^{-1}$

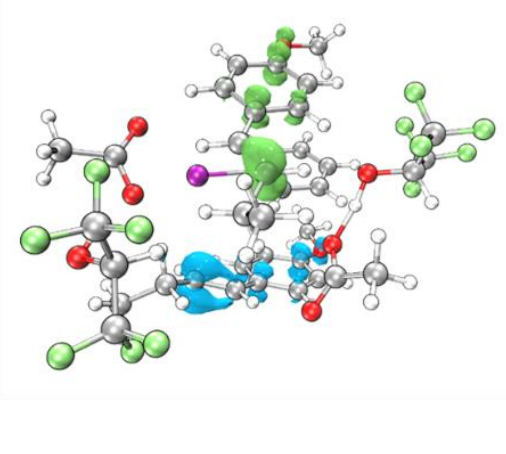

Figure 8. The geometries and spin density isosurfaces (with isovalue of 0.01) of the possible intermediates for the overall 2e-transfer between PIDA HFIP $_{\text {and }}$ 4. Energies for the triplet state are shown in brackets. Bond lengths are in $\AA$.

In a summary, the seemingly similar overall 2e-transfer reactions involving PIDA $\mathbf{A F I P}_{\text {and }}$ DMP $\mathbf{P F I P}_{\text {are }}$ different in nature. Although both of them are initialized by the energetically-favored trimer formation, the electron transfer is concerted for $\mathbf{D M P}_{\mathbf{H F I P}}$ and stepwise for $\mathbf{P I D A}_{\mathbf{H F I P}}$, as suggested by our results.

\section{Molecular dynamics of HETD and HOMD}

In general, the initiative SEO step from styrene 4 is shown to be more reactive with DMP catalyst since the SEO occurs with the more deficient catalyst $\mathrm{I}(\mathrm{V})(\mathrm{LUMO}=-1.56 \mathrm{eV})$ over less deficient one $\mathrm{I}(\mathrm{III})(\mathrm{LUMO}=$ 
$-0.73 \mathrm{eV}$ ) (see Figure S14), apparently indicating that the initiation in HETD is faster than HOMD under DMP conditions. However, and from a synthetically perspective point of view, there is a relative competition between both processes which is experimentally seen (see supporting information for ref 41 ). ${ }^{41}$ The calculations indicate that once the radical cation $\mathbf{4}^{+}$is formed, entering homo [2+2] cycloaddition is relatively possible. The results above (Figure 4 and 5) reveal that the HOMD starts with a barrier of $9.5 \mathrm{kcal} \mathrm{mol}^{-1}$ via TS $\mathbf{1 1}^{+}$whereas HETD starts with lower barriers of $8.3 \mathrm{kcal} \mathrm{mol}^{-1}$ via TS $\mathbf{8}^{+}$, implying a barrier difference of $\Delta \Delta \mathrm{G}^{\ddagger}=1.2 \mathrm{kcal} \mathrm{mol}^{-1}$. This refers to that the HETD is comparingly predominant. This is in general good agreement with the experimental findings, in which the HETD reaction proceeds an equivalent ratio of styrenes 5 to 4 of $2: 1$. A further evidence for the competition between HOMD and HETD is emerged form QCT molecular dynamics of the first $\mathrm{C}-\mathrm{C}$ bond formation (shown below).

Quasiclassical trajectory molecular dynamics (QCTMD) simulations were utilized to understand the chronological character for formation of first $\mathrm{C}-\mathrm{C}$ bonds in the HETD and HOMD (Figure 7). ${ }^{62-68}$ The QCTMD simulations were carried out using the PROGDYN program, ${ }^{69}$ a script suite that works in combination with Gaussian 09. 44 and 63 trajectories were generated starting from the TSs $\mathbf{8}^{+}$and $\mathbf{1 1}^{+}$, respectively, in which forward and backward propagations ( $\mathrm{t}=0 \mathrm{fs}$ ) are initiated showing the typical reactive bonds toward either cationic uncyclized intermediates $\left(9^{+}\right.$and $\mathbf{1 2}^{+}$) or reactants (styrene $\mathbf{4}^{+}, \mathbf{4}$ and $\mathbf{5}$ ). No recrossing is observed in our simulation. The $\mathrm{C} 3-\mathrm{C} 4$ distance is rapidly shortened to $\sim 1.6 \AA$ in most trajectories, and the bond remains in the whole trajectory once formed although for a small proportion of trajectories the C3-C4 distance oscillates in the range between $1.6 \AA$ and $2.0 \AA$. By recording the timing for the $\mathrm{C} 3-\mathrm{C} 4$ distance to be shortened below 1.6 $\AA$, we obtained the average timing for the first $\mathrm{C}-\mathrm{C}$ bond formation at 43.0 and 47.0 fs for HETD $\mathbf{8}^{+}$and HOMD $\mathbf{1 1}^{+}$, respectively. It is interesting that although the average timing is similar, there are more trajectories exhibiting larger timing for $\mathrm{C}-\mathrm{C}$ bond formation for the HOMD pathway, which may indicate a flatter potential energy surface in the post-transition state period. Comparison of the average time for the first $\mathrm{C}-\mathrm{C}$ bond formation through TS $\mathbf{8}^{+}$and TS $\mathbf{1 1}^{+}$reveals a short timing gap of 4.0 fs. Also, the timing for first $\mathrm{C}-\mathrm{C}$ bond formation for the unstacked TSs were obtained and shown a short timing gap where 46 fs for HOMD, derived from 24 trajectories, and 44 fs for HETD, derived from 20 trajectories (see Figure S8). Overall, the very small timing gap between both pathways explains that HETD and HOMD are dynamically competitive. 

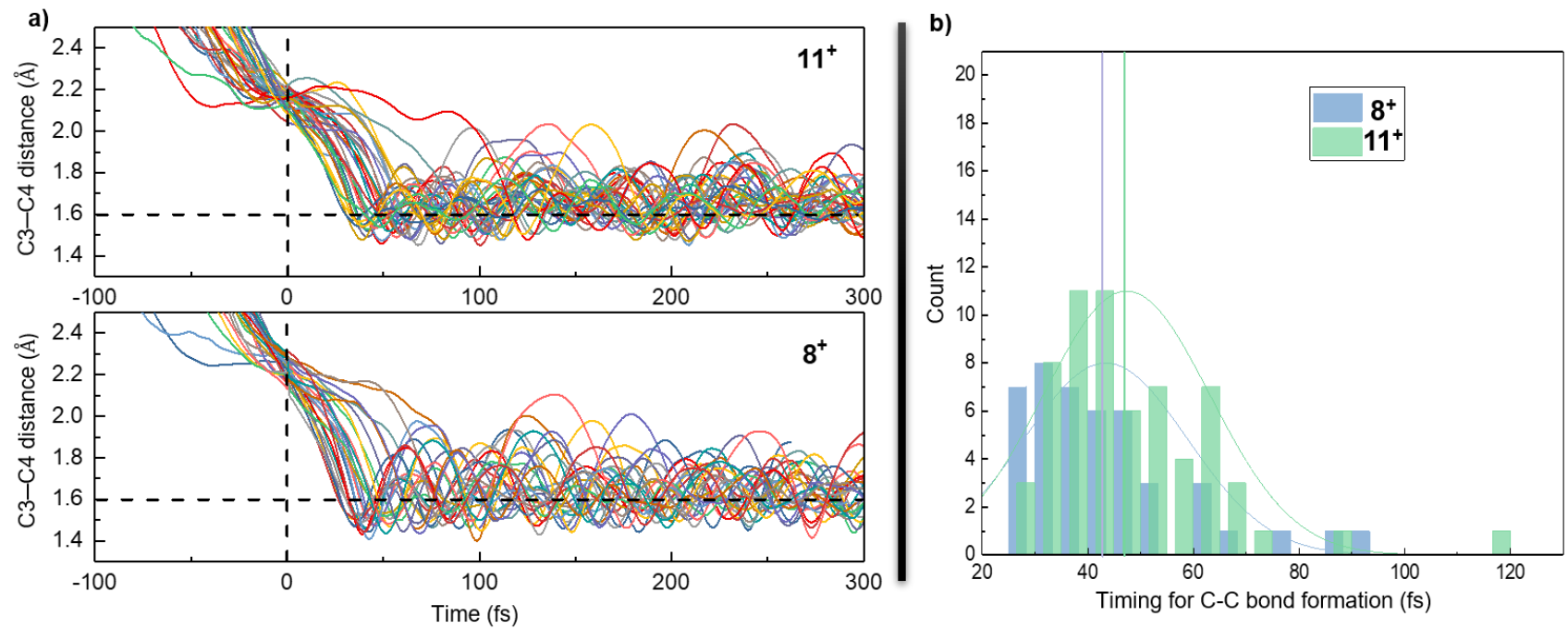

Figure 7. (a) Evolution of the $\mathrm{C}-\mathrm{C}$ distance corresponding to the first $\mathrm{C}-\mathrm{C}$ bond formation along quasiclassical trajectories initialized from TSs $\mathbf{8}^{+}$and $\mathbf{1 1}^{+}$calculated by the $w \mathrm{~B} 97 \mathrm{XD} / 6-31 \mathrm{G}(\mathrm{d})$ level of theory. All trajectories start from the initial geometry ( $\mathrm{t}=0 \mathrm{fs})$ generated by adding a random displacement to the transition state, and both directions are shown in positive and negative part of the horizontal axis. (b) A histogram for the $\mathrm{C}-\mathrm{C}$ bond formation timing, where the average timing for each reaction is shown by the vertical line.

\section{Conclusions}

DFT calculations at the (SMD)-wB97XD/Def2-TZVPP,6-311+G(d,p)//wB97XD/6-31G(d),LANL2DZ level of theory were exploited to provide mechanistic insights into the HVIR-promoted hetero- and homodimerizations of styrenes facilitated by HFIP. The computational level was validated through comparison between calculated and measured redox potentials of different substituted trans- $\beta$-methylstyrenes. The findings achieved in this study can be summarized as follows. First, the hypervalent iodine-mediated hetero- and homodimerizations of styrenes initiated with two-electron reductive cleavage of two $\mathrm{I}-\mathrm{O}$ bond cleavages giving iodinane $\mathrm{I}(\mathrm{III})$ and iodobenzene I(I) when DMP and PIDA are used, respectively, plus two acetate groups stabilized by strong hydrogen bonding interactions provided by HFIP. Accordingly, the propagation of the reaction is accomplished by radically-cationic hetero- and homodimerized cyclic intermediates. However, a disfavored initiation via a one-electron reduction was found, especially for the HOMD in the presence of PIDA where initiation become highly unlikely to take place. This is in disagreement with the mild experimental conditions and, therefore, two- 
electron process is required to initiate the radically-cationic [2+2] cycloaddition reaction. Also, the change in oxidation state reported here, $\mathrm{I}(\mathrm{V}) \rightarrow \mathrm{I}(\mathrm{III})$ and $\mathrm{I}(\mathrm{III}) \rightarrow \mathrm{I}(\mathrm{I})$, are commensurate with the oxidation of alcohols by hypervalent iodine reagents. By further investigation of the nature of the overall two-electron transfer, PIDA and DMP were shown to follow different reaction pathways for the overall two-electron transfer: the two electron transfer events are concerted for DMP and accompanied by the cleavage of one I-O bond, whereas are stepwise for PIDA, although for both oxidants occur within a tri-molecular complex stabilized by pi-stacking. Second, the mechanism of HETD and HOMD is a radically-characterized $\pi-\pi$ stacked head-to-head stepwise [2+2] cycloaddition initiated via SEO by DMP and PIDA, respectively. DFT results supported by quasiclassical molecular dynamics simulations show that HOMD is dynamically competing pathway to HETD although the latter is relatively faster, in accordance with experimental observations. Third, the rate-determining step was evaluated to be critical with I(III) to initiate the reaction as a thermodynamically endergonic whereas endergonicity from I(V) initiation showed to be very modest. The initiative SEO step was computed to be more reactive with DMP catalyst as the SEO occurs with the more deficient catalyst I(V) (lower LUMO) over less deficient one I(III) (higher LUMO).

Overall, this mechanistic study brings significantly important insights into a such influential synthetic utility and opens possibilities toward advancing an efficient protocol for stereoselective approaches of simple and complex hetero- and homodimerizations. We envision that using DFT simulations on catalyzed SEO will enhance and warrant further attentions toward developing various oxidants to synthetically access a wide range of substrates used for bioactive and synthetic cyclobutane-containing products in a more efficiently-controlled fashion.

\section{ASSOCIATED CONTENT}

\section{Supporting Information}

The Supporting Information is available free of charge on the ACS Publications website. Further computational results, absolute energies and cartesian coordinates (PDF) 


\section{AUTHOR INFORMATION}

\section{Corresponding Author}

Aqeel A. Hussein - School of Chemistry, University of Southampton, Southampton, Hampshire, SO17 1BJ, United Kingdom; College of Dentistry, University of Al-Ameed, Karbala PO Box 198, Iraq; orcid.org/00000002-9259-9609; Email: aahh1f19@ soton.ac.uk

\section{Authors}

Yumiao Ma - BSJ Institute, Haidian, Beijing, 100084, People's Republic of China; orcid.org/0000-0002$0628-8864$

Ahmed Al-Yasari - School of Chemistry, University of East Anglia, Norwich, NR4 7TJ, United Kingdom; Department of Chemistry, Faculty of Sciences, University of Kerbala, Kerbala, Iraq; orcid.org/0000-0001$8768-1248$

\section{Notes}

The authors declare no competing financial interest.

\section{Acknowledgements}

The authors acknowledge the computational resources from the iridis 4 supercomputer supported by the University of Southampton. A.A.H. highly acknowledge the University of Southampton/School of Chemistry for providing the visitor-status research position (2717441/EB00-VISIT). A.A.H. thanks Prof. Richard C. D. Brown for his valuable support.

\section{References}

1. Xu, Y.; Conner, M. L.; Brown, M. K. Cyclobutane and Cyclobutene Synthesis: Catalytic Enantioselective 2+2 Cycloadditions. Angew. Chem. Int. Ed. 2015, 54, 11918-11928.

2. Margaretha, P. Retrospective View on Recent Developments in Cyclobutane Synthesis via [2+2] Photocycloaddition of Unsaturated Ketones to Acyclic Dienes. Helv. Chim. Acta 2014, 1027-1035. 
3. Dembitsky, V. M. Naturally occurring bioactive Cyclobutane-containing (CBC) alkaloids in fungi, fungal endophytes, and plants. Phytomedicine 2014, 21, 1559-1581.

4. Dembitsky, V. M. Bioactive cyclobutane-containing alkaloids. J. Nat. Med. 2008, 62, 1-33.

5. Cretton, S.; Bartholomeusz, T. A.; Jeannerat, D.; Munoz, O.; Christen, P.; Hostettmann, K. New cyclobutane-containing tropane alkaloids from the aerial parts of Schizanthus grahamii. Planta Med. 2009, 75, 916-916.

6. Ito, H.; Toyoda, T.; Sawamura, M. Stereospecific Synthesis of Cyclobutylboronates through Copper(I)Catalyzed Reaction of Homoallylic Sulfonates and a Diboron Derivative. J. Am. Chem. Soc. 2010, 132, 5990-5992.

7. Noucti, N. N.; Alexanian, E. J. Stereoselective Nickel- Catalyzed 2+2 Cycloadditions of EneAllenes. Angew. Chem. Int. Ed. 2015, 54, 5447-5450.

8. Gulías, M.; Collado, A.; Trillo, B.; López, F.; Oñate, E.; Esteruelas, M. A.; Mascareñas, J. L. Ruthenium-Catalyzed $(2+2)$ Intramolecular Cycloaddition of Allenenes. J. Am. Chem. Soc. 2011, 133, 7660-7663.

9. Luzung, M. R.; Mauleón, P.; Toste, F. D. Gold(I)-Catalyzed [2 + 2]-Cycloaddition of Allenenes. J. Am. Chem. Soc. 2007, 129, 12402-12403.

10. Chiba, K.; Miura, T.; Kim, S.; Kitano, Y.; Tada, M. Electrocatalytic Intermolecular Olefin CrossCoupling by Anodically Induced Formal [2+2] Cycloaddition between Enol Ethers and Alkenes. J. Am. Chem. Soc. 2001, 123, 11314-11315.

11. Rath, B. B.; Kole, G. K.; Morris, S. A.; Vittal, J. J. Rotation of a helical coordination polymer by mechanical grinding. Chem. Commun. 2020.

12. Ledwith, A. Cation radicals in electron transfer reactions. Acc. Chem. Res. 1972, 5, 133-139.

13. Bell, F. A.; Crellin, R. A.; Fujii, H.; Ledwith, A. Cation-radicals: metal-catalysed cyclodimerisation of aromatic enamines. J. Chem. Soc. D: Chem. Commun. 1969, 251-252.

14. Bauld, N. L.; Bellville, D. J.; Harirchian, B.; Lorenz, K. T.; Pabon, R. A.; Reynolds, D. W.; Wirth, D. D.; Chiou, H. S.; Marsh, B. K., Cation radical pericyclic reactions. Acc. Chem. Res. 1987, 20, 371-378.

15. Bauld, N. L.; Bellville, D. J.; Pabon, R.; Chelsky, R.; Green, G. Theory of cation-radical pericyclic reactions. J. Am. Chem. Soc. 1983, 105, 2378-2382. 
16. Bauld, N. L.; Pabon, R. Cation radical catalyzed olefin cyclodimerization. J. Am. Chem. Soc. 1983, 105, 633-634.

17. Ischay, M. A.; Ament, M. S.; Yoon, T. P. Crossed intermolecular [2 + 2] cycloaddition of styrenes by visible light photocatalysis. Chem. Sci. 2012, 3, 2807-2811.

18. Riener, M.; Nicewicz, D. A. Synthesis of cyclobutane lignans via an organic single electron oxidantelectron relay system. Chem. Sci. 2013, 4, 2625-2629.

19. Jiang, Y.; Wang, C.; Rogers, C. R.; Kodaimati, M. S.; Weiss, E. A. Regio- and diastereoselective intermolecular [2+2] cycloadditions photocatalysed by quantum dots. Nat. Chem. 2019, 11, 1034-1040.

20. Ischay, M. A.; Lu, Z.; Yoon, T. P. [2+2] Cycloadditions by Oxidative Visible Light Photocatalysis. J. Am. Chem. Soc. 2010, 132, 8572-8574.

21. Lu, Z;; Yoon, T. P., Visible Light Photocatalysis of [2+2] Styrene Cycloadditions by Energy Transfer. Angew. Chem. Int. Ed. 2012, 51, 10329-10332.

22. Hurtley, A. E.; Lu, Z.; Yoon, T. P. [2+2] Cycloaddition of 1,3-Dienes by Visible Light Photocatalysis. Angew. Chem. Int. Ed. 2014, 53, 8991-8994.

23. Fleming, S. A.; Ward, S. C. Stereocontrolled photochemical [2+2] cycloaddition. Tetrahedron Lett. 1992, 33, 1013-1016.

24. Ward, S. C.; Fleming, S. A. [2 + 2] Photocycloaddition of Cinnamyloxy Silanes. J. Org. Chem. 1994, 59, 6476-6479.

25. Horibe, T.; Katagiri, K.; Ishihara, K. Radical-Cation-Induced Crossed [2+2] Cycloaddition of ElectronDeficient Anetholes Initiated by Iron(III) Salt. Adv. Synth. Catal. 2020, 362, 960-963.

26. Zhao, L.-M.; Lei, T.; Liao, R.-Z.; Xiao, H.; Chen, B.; Ramamurthy, V.; Tung, C.-H.; Wu, L.-Z. VisibleLight-Triggered Selective Intermolecular [2+2] Cycloaddition of Extended Enones: 2-Oxo-3-enoates and 2,4Dien-1-ones with Olefins. J. Org. Chem. 2019, 84, 9257-9269.

27. Li, R.; Ma, B. C.; Huang, W.; Wang, L.; Wang, D.; Lu, H.; Landfester, K.; Zhang, K. A. I. Photocatalytic Regioselective and Stereoselective $[2+2]$ Cycloaddition of Styrene Derivatives Using a Heterogeneous Organic Photocatalyst. ACS Catal. 2017, 7, 3097-3101. 
28. Amjaour, H.; Wang, Z.; Mabin, M.; Puttkammer, J.; Busch, S.; Chu, Q. R. Scalable preparation and property investigation of a cis-cyclobutane-1,2-dicarboxylic acid from $\beta$-trans-cinnamic acid. Chem. Commun. 2019, 55, 214-217.

29. Yoshimura, A.; Zhdankin, V. V. Advances in Synthetic Applications of Hypervalent Iodine Compounds. Chem. Rev. 2016, 116, 3328-3435.

30. Li, Y.; Hari, D. P.; Vita, M. V.; Waser, J. Cyclic Hypervalent Iodine Reagents for Atom-Transfer Reactions: Beyond Trifluoromethylation. Angew. Chem. Int. Ed. 2016, 55, 4436-4454.

31. Colomer, I.; Chamberlain, A. E. R.; Haughey, M. B.; Donohoe, T. J. Hexafluoroisopropanol as a highly versatile solvent. Nat. Rev. Chem. 2017, 1, 0088.

32. Zhu, Y.; Colomer, I.; Thompson, A. L.; Donohoe, T. J. HFIP Solvent Enables Alcohols To Act as Alkylating Agents in Stereoselective Heterocyclization. J. Am. Chem. Soc. 2019, 141, 6489-6493.

33. Colomer, I.; Barcelos, R. C.; Christensen, K. E.; Donohoe, T. J. Orthogonally Protected 1,2-Diols from Electron-Rich Alkenes Using Metal-Free Olefin syn-Dihydroxylation. Org. Lett. 2016, 18, 5880-5883.

34. Raheem, I. T.; Thiara, P. S.; Peterson, E. A.; Jacobsen, E. N. Enantioselective Pictet-Spengler-Type Cyclizations of Hydroxylactams: H-Bond Donor Catalysis by Anion Binding. J. Am. Chem. Soc. 2007, 129, 13404-13405.

35. Lv, H.; Zhan, J.-H.; Cai, Y.-B.; Yu, Y.; Wang, B.; Zhang, J.-L. $\pi-\pi$ Interaction Assisted Hydrodefluorination of Perfluoroarenes by Gold Hydride: A Case of Synergistic Effect on C-F Bond Activation. J. Am. Chem. Soc. 2012, 134, 16216-16227.

36. Doyle, A. G.; Jacobsen, E. N. Small-Molecule H-Bond Donors in Asymmetric Catalysis. Chem. Rev. 2007, 107, 5713-5743.

37. Nicolaou, K. C.; Baran, P. S.; Zhong, Y.-L.; Vega, J. A., Novel IBX-Mediated Processes for the Synthesis of Amino Sugars and Libraries Thereof. Angew. Chem. Int. Ed. 2000, 39, 2525-2529.

38. Nicolaou, K. C.; Baran, P. S.; Zhong, Y. L.; Barluenga, S.; Hunt, K. W.; Kranich, R.; Vega, J. A. Iodine(V) Reagents in Organic Synthesis. Part 3. New Routes to Heterocyclic Compounds via o-Iodoxybenzoic Acid-Mediated Cyclizations: Generality, Scope, and Mechanism. J. Am. Chem. Soc. 2002, 124, 2233-2244.

39. Nicolaou, K. C.; Baran, P. S.; Kranich, R.; Zhong, Y.-L.; Sugita, K.; Zou, N. Mechanistic Studies of Periodinane-Mediated Reactions of Anilides and Related Systems. Angew. Chem. Int. Ed. 2001, 40, 202-206. 
40. Janza, B.; Studer, A. Stereoselective Cyclization Reactions of IBX-Generated Alkoxyamidyl Radicals. J. Org. Chem. 2005, 70, 6991-6994.

41. Colomer, I.; Coura Barcelos, R.; Donohoe, T. J. Catalytic Hypervalent Iodine Promoters Lead to Styrene Dimerization and the Formation of Tri- and Tetrasubstituted Cyclobutanes. Angew. Chem. Int. Ed. 2016, $55(15), 4748-4752$.

42. Zhu, Y.; Colomer, I.; Donohoe, T. J. Hypervalent iodine initiated intramolecular alkene dimerisation: a stereodivergent entry to cyclobutanes. Chem. Commun. 2019, 55, 10316-10319.

43. Colomer, I.; Batchelor-McAuley, C.; Odell, B.; Donohoe, T. J.; Compton, R. G. Hydrogen Bonding to Hexafluoroisopropanol Controls the Oxidative Strength of Hypervalent Iodine Reagents. J. Am. Chem. Soc. 2016, $138,8855-8861$.

44. Farshadfar, K.; Chipman, A.; Yates, B. F.; Ariafard, A. DFT Mechanistic Investigation into BF3Catalyzed Alcohol Oxidation by a Hypervalent Iodine(III) Compound. ACS Catal. 2019, 9, 6510-6521.

45. Ganji, B.; Ariafard, A. DFT mechanistic investigation into phenol dearomatization mediated by an iodine(iii) reagent. Org. Biomol. Chem. 2019, 17, 3521-3528.

46. Kaur, A.; Ariafard, A., Mechanistic investigation into phenol oxidation by IBX elucidated by DFT calculations. Org. Biomol. Chem. 2020, 18, 1117-1129.

47. De Munari, S.; Frigerio, M.; Santagostino, M. Hypervalent Iodine Oxidants: Structure and Kinetics of the Reactive Intermediates in the Oxidation of Alcohols and 1,2-Diols by o-Iodoxybenzoic Acid (IBX) and Dess-Martin Periodinane. A Comparative 1H-NMR Study. J. Org. Chem. 1996, 61, 9272-9279.

48. O'Nei, L. L.; Wiest, O. Acyclic or Long-Bond Intermediate in the Electron-Transfer-Catalyzed Dimerization of 4-Methoxystyrene. J. Org. Chem. 2006, 71, 8926-8933.

49. Guo, C.; Cui, L.; Chen, B.; Yuan, J.; Tian, Z. A theoretical study on the stereoconvergency of the intramolecular radical cation [2+2] cycloadditions of bis(styrenes). RSC Adv. 2012, 2, 9932-9937.

50. Li, Y.; Guo, C.; Chen, B.-Z. A theoretical study on intermolecular [2+2] radical cation cycloaddition reactions and the competition between concerted and stepwise mechanisms. Comput. Theor. Chem. 2016, 1078, $163-172$.

51. An initial version of this work was deposited in ChemRxiv on 08/20/2020, Reference (Insert DOI) (https://chemrxiv.org/ndownloader/files/24120110, https://chemrxiv.org/ndownloader/files/24120119). 
52. Chai, J.-D.; Head-Gordon, M. Long-range corrected hybrid density functionals with damped atom-atom dispersion corrections. Phys. Chem. Chem. Phys. 2008, 10, 6615-6620.

53. Nelsen, S. F.; Blackstock, S. C.; Kim, Y. Estimation of inner shell Marcus terms for amino nitrogen compounds by molecular orbital calculations. J. Am. Chem. Soc. 1987, 109, 677-682.

54. Vaissier, V.; Barnes, P.; Kirkpatrick, J.; Nelson, J. Influence of polar medium on the reorganization energy of charge transfer between dyes in a dye sensitized film. Phys. Chem. Chem. Phys. 2013, 15, 4804-4814. 55. Manke, F.; Frost, J. M.; Vaissier, V.; Nelson, J.; Barnes, P. R. F., Influence of a nearby substrate on the reorganization energy of hole exchange between dye molecules. Phys. Chem. Chem. Phys. 2015, 17 (11), 73457354.

56. Meyer, S. D.; Schreiber, S. L. Acceleration of the Dess-Martin Oxidation by Water. J. Org. Chem. 1994, $59,7549-7552$.

57. Dess, D. B.; Martin, J. C. A useful 12-I-5 triacetoxyperiodinane (the Dess-Martin periodinane) for the selective oxidation of primary or secondary alcohols and a variety of related 12-I-5 species. J. Am. Chem. Soc. 1991, 113, 7277-7287.

58. Dess, D. B.; Martin, J. C. Readily accessible 12-I-5 oxidant for the conversion of primary and secondary alcohols to aldehydes and ketones. J. Org. Chem. 1983, 48, 4155-4156.

59. Demaille, C.; Bard, A. J. Kinetics and mechanism of the anodic dimerization of trans-anethole studied by cyclic voltammetry and scanning electrochemical microscopy. Acta Chem. Scan. 1999, 53, 842-848.

60. Johnson, E. R.; Keinan, S.; Mori-Sánchez, P.; Contreras-García, J.; Cohen, A. J.; Yang, W. Revealing Noncovalent Interactions. J. Am. Chem. Soc. 2010, 132, 6498-6506.

61. Kokkinidis, G.; Hatzigrigoriou, E.; Sazou, D.; Varvoglis, A. Electrochemical reduction of some hypervalent iodine compounds. Electrochim. Acta 1991, 36, 1391-1395.

62. Burns, J. M.; Boittier, E. D. Pathway Bifurcation in the $(4+3) /(5+2)$-Cycloaddition of Butadiene and Oxidopyrylium Ylides: The Significance of Molecular Orbital Isosymmetry. J. Org. Chem. 2019, 84, 59976005

63. Villar López, R.; Faza, O. N.; Silva López, C. Dynamic Effects Responsible for High Selectivity in a [3,3] Sigmatropic Rearrangement Featuring a Bispericyclic Transition State. J. Org. Chem. 2017, 82, $4758-4765$. 
64. Patel, A.; Chen, Z.; Yang, Z. Y.; Gutierrez, O.; Liu, H. W.; Houk, K. N.; Singleton, D. A. Dynamically Complex 6+4 and 4+2 Cycloadditions in the Biosynthesis of Spinosyn A. J. Am. Chem. Soc. 2016, 138, $3631-3634$.

65. Xu, L.; Doubleday, C. E.; Houk, K. N. Dynamics of Carbene Cycloadditions. J. Am. Chem. Soc. 2011, $133,17848-17854$.

66. Xu, L.; Doubleday, C. E.; Houk, K. N. Dynamics of 1,3-Dipolar Cycloadditions: Energy Partitioning of Reactants and Quantitation of Synchronicity. J. Am. Chem. Soc. 2010, 132, 3029-3037.

67. Black, K.; Liu, P.; Xu, L.; Doubleday, C.; Houk, K. N. Dynamics, transition states, and timing of bond formation in Diels-Alder reactions. Proc. Natl. Acad. Sci. U.S.A. 2012, 109, 12860-12865.

68. Patel, A.; Chen, Z.; Yang, Z.; Gutiérrez, O.; Liu, H.-w.; Houk, K. N.; Singleton, D. A. Dynamically Complex [6+4] and [4+2] Cycloadditions in the Biosynthesis of Spinosyn A. J. Am. Chem. Soc. 2016, 138, 3631-3634.

69. Singleton, D. A.; Hang, C.; Szymanski, M. J.; Greenwald, E. E. A New Form of Kinetic Isotope Effect. Dynamic Effects on Isotopic Selectivity and Regioselectivity. J. Am. Chem. Soc. 2003, 125, 1176-1177. 